\title{
El impacto del software libre en el sector empresarial de Bogotá.
}

Lugo Manuel Barbosa Guerrero ${ }^{1}$

\section{Resumen}

Este articulo presenta los resultados de un proyecto de investigación que evaluó el uso del software libre en una muestra de 50 empresas entre pymes y grandes empresas en Bogotá. Se encontró que el 47.62\% de la muestra estudiada utiliza el software libre, mientras que el $52.38 \%$ no lo utiliza.

Palabras clave: software libre, utilidad, sistema operativo, software propietario, Linux

\section{The impact of freeware in Bogotá's business sector}

\begin{abstract}
This article presents the results of a research project that assessed the use free software in a sample of 50 businesses, a mix of small, medium and large companies in Bogotá. Findings are that $47.62 \%$ of the businesses in the survey use free software, while the remaining $52.38 \%$ do not use it.
\end{abstract}


Key words: freeware, utility, operating system, proprietary software, Linux.

Recibido: 26 de julio 2012

Aceptado: 22 de noviembre 2012

\section{Introducción}

Estudios realizados en España reflejan que el $80 \%$ de pymes y el 63\% de grandes empresas valoran el uso de software libre en su estrategia tecnológica. Mientras que un $90 \%$ elijen software libre por el bajo o nulo costo de licencia, el acceso al código fuente es razón para la elección en el $80 \%$ de las mismas.

A nivel internacional, Europa es pionera en el desarrollo de software libre, que alcanza ya el $29 \%$ de todo el software desarrollado ${ }^{2}$. El $65 \%$ de las empresas han adoptado o tienen interés en adoptar software de fuentes abiertas. De ellas, un 53\% tienen software libre para la gestión de bases de datos, un 32\% para la gestión de redes, y un $30 \%$ en seguridad. A futuro, la evolución prevista es en e-learning, gestión de redes, desarrollo de aplicaciones y de creación de contenidos digitales.

En España, el 33\% del software utilizado por las pymes es libre y es principalmente utilizado para sistemas de gestión de bases de datos (66\%), servidores web (61\%), sistemas operativos (61\%) y suites ofimáticas (48\%). En el caso de las grandes empresas, este porcentaje es del 14\%, principalmente usado en servidores web $(50 \%)$, sistemas operativos $(47 \%)$, gestión de bases de datos $(41 \%)$ y suites ofimáticas $(28 \%)^{3}$.

Las razones para elegir software libre en las grandes corporaciones son el menor o nulo costo de licencia (91\% de las empresas encuestadas), el acceso al código fuente $(80 \%)$, el menor costo total de propiedad TCO (78\%), y la valoración de los estándares y procesos de desarrollo abierto (72\%). En cuanto a las pymes, igualmente el menor o nulo costo de licencia es el principal valor (80\%), seguido del acceso al código fuente (77\%), el menor TCO (79\%), y los estándares y procesos de desarrollo abiertos $(77 \%)$.

El $59 \%$ de las pymes, y el $41 \%$ de las grandes empresas que han adoptado alguna solución de software libre reconocen haber reducido costos, y se muestran muy satisfechas con su uso el $82 \%$ de grandes empresas y el $78 \%$ de las pymes. Finalmente, entre las empresas usuarias, el $85 \%$ de las pymes y el $95 \%$ de las grandes compañías afirman hacer aportaciones a la comunidad de desarrollo.

2 Tomado el 22 de noviembre de 2011 de: http://www.cenatic.es/

3 Tomado el 2 de noviembre de 2011 de: http://www.mentatconsultores.com/?s=software+libre\&searchsubmit= 
Por otra parte, mientras Estados Unidos lidera en términos de grandes negocios relacionados con software libre, en Colombia un gran porcentaje de empresas privadas y del sector público ya vienen haciendo uso del software libre con algún tipo de aplicación. En el sector público, Europa tiene una mayor penetración, aunque podría ser alcanzada pronto por Asia y Latinoamérica.

Se ha comprobado según estudios realizados (Cámara de comercio, International Data Corporation -IDC), que en Colombia solo el 32.2\% de las empresas invierte en tecnología, un porcentaje muy bajo que dificulta el uso tecnológico en el sector empresarial y afecta el crecimiento en el uso del software libre.

Conocer las características de estructura y estructuras económicas de las empresas evaluadas, también conocer sus beneficios, oportunidades y límites al implementar el software libre.

\section{Materiales y métodos}

Muestra: Se utilizó una muestra de 50 empresas en la ciudad de Bogotá, Tabla 1

Tabla 1. Empresas participantes en el estudio

\begin{tabular}{|c|c|c|}
\hline No. & Organización & Sector \\
\hline 1 & $\begin{array}{l}\text { AGENCIA DE ADUANAS TECTCOMEX } \\
\text { SAS }\end{array}$ & Agencia Aduanera. \\
\hline 2 & ALLUS GLOBAL BPO CENTER & $\begin{array}{c}\text { Compañía líder en América Latina en la } \\
\text { provisión de soluciones BPO. }\end{array}$ \\
\hline 3 & ASTRO'S SPORT & Creación implementos deportivos. \\
\hline 4 & AVIATUR SA & Agencia de viajes y turismo. \\
\hline 5 & BANCO AGRARIO DE COLOMBIA & Servicios bancarios. \\
\hline 6 & BANCO AV VILLAS & Servicios bancarios. \\
\hline 7 & BANCO CAJA SOCIAL & Servicios bancarios. \\
\hline 8 & BANCO DE BOGOTÁ & Servicios bancarios. \\
\hline 9 & BANCO DE LA REPUBLICA & Política monetaria y servicios bancarios. \\
\hline 10 & BBVA SEGUROS & Servicios bancarios. \\
\hline 11 & BELLA PIEL S.A. & Fabricación de artículos de cuero. \\
\hline 12 & CIVILTEC LTDA & Empresa de ingeniería civil. \\
\hline 13 & CLINICA SHAIO & Servicios médicos. \\
\hline
\end{tabular}




\begin{tabular}{|c|c|c|}
\hline No. & Organización & Sector \\
\hline 14 & COBRANZA EFECTIVA LTDA & $\begin{array}{l}\text { Información comercial, financiera y de } \\
\text { marketing. }\end{array}$ \\
\hline 15 & COMERCIALIZADORA BALDINISA & Comercio de artículos de cuero. \\
\hline 16 & CONIAC CENTER AMERICAS & Producción industrial. \\
\hline 17 & CONSTRUCTORA BOLIVAR & Proyectos de vivienda. \\
\hline 18 & COOMEVA SECTOR SALUD & Servicios médicos. \\
\hline 19 & CORP. EDUCATIVA INDOAMERICANA & $\begin{array}{c}\text { Escuela de capacitación técnica en } \\
\text { aviación. }\end{array}$ \\
\hline 20 & EDUARDO L GERLEIN S.A. & Agentes de carga. \\
\hline 21 & FINANCIERA JURISCOOP & $\begin{array}{l}\text { Financiamiento comercial - cooperativa } \\
\text { financiera. }\end{array}$ \\
\hline 22 & GARCIA VARGAS INGENIEROS LTDA & $\begin{array}{c}\text { Diseño, fabricación y automatización } \\
\text { industrial. }\end{array}$ \\
\hline 23 & GAS TECHNOLOGY COLOMBIA & Compañía de energía Colombiana. \\
\hline 24 & GDF SUEZ ENERGY COLOMBIA LTDA & Compañía de energía Colombiana. \\
\hline 25 & $\begin{array}{l}\text { GENERAL DE EQUIPOS DE COLOMBIA } \\
\text { S A }\end{array}$ & $\begin{array}{l}\text { Mantenimiento de maquinaria de } \\
\text { construcción. }\end{array}$ \\
\hline 26 & GRUPO COREMAR & $\begin{array}{c}\text { Empresa de infraestructura marítima y } \\
\text { portuaria. }\end{array}$ \\
\hline 27 & HELM BANK & Servicios bancarios. \\
\hline 28 & HOSPITAL LA VICTORIA & Servicios médicos. \\
\hline 29 & IDIPRON & $\begin{array}{c}\text { Instituto Distrital para la Protección de la } \\
\text { Niñez y la Juventud }\end{array}$ \\
\hline 30 & $\begin{array}{l}\text { INSTITUTO NACIONAL DE } \\
\text { CANCEROLOGIA }\end{array}$ & Servicios médicos. \\
\hline 31 & INTERAUDIT & Auditores de estados financieros. \\
\hline 32 & LA EQUIDAD SEGUROS & Aseguradora cooperativa. \\
\hline 33 & COOMAYORCUN & Sector cooperativo. \\
\hline 34 & MEGAPLAST LIMITADA & $\begin{array}{l}\text { Fabricación de piezas técnicas para la } \\
\text { industria. }\end{array}$ \\
\hline 35 & $\begin{array}{l}\text { MTI- MANEJO TECNICO DE } \\
\text { INFORMACION }\end{array}$ & $\begin{array}{l}\text { Administradores de procesos de } \\
\text { información. }\end{array}$ \\
\hline 36 & OPCIONES ADMINISTRATIVAS & Economías de escala. \\
\hline 37 & PANADERIAS PACOLOMBIA & Sector alimentos. \\
\hline 38 & PRICE WATER HOUSE COOPERS AG & Asesores Gerenciales Asociados. \\
\hline 39 & $\begin{array}{c}\text { RECURSOS HUMANOS EXCLUSIVOS } \\
\text { S.A. }\end{array}$ & Empresa de servicios temporales. \\
\hline 40 & $\begin{array}{l}\text { SECRETARIA DE EDUCACION } \\
\text { DISTRITAL }\end{array}$ & $\begin{array}{l}\text { Rectora de la educación en el Distrito } \\
\text { Capital. }\end{array}$ \\
\hline
\end{tabular}


Revista de

investigaciones UNAD

Volumen 11. Número 2. Julio 2012

\begin{tabular}{|c|c|c|}
\hline No. & Organización & Sector \\
\hline 41 & SEDEL & Control integral de plagas y desinfección. \\
\hline 42 & SODIMAC COLOMBIA S.A. & Productos para el hogar. \\
\hline 43 & TELEPERFORMANCE S.A. & $\begin{array}{l}\text { Prestadora de servicios de contact } \\
\text { center y BPO. }\end{array}$ \\
\hline 44 & $\begin{array}{c}\text { TURISMO EN CASAS Y FINCAS } \\
\text { SATUCASA }\end{array}$ & Inmobiliarias y turismo. \\
\hline 45 & UNIANDES & Institución de Educación superior. \\
\hline 46 & $\begin{array}{l}\text { UNIVERSIDAD COOPERATIVA DE } \\
\text { COLOMBIA }\end{array}$ & Institución de Educación superior. \\
\hline 47 & VAS COLOMBIA S.A. & $\begin{array}{l}\text { Importadora de automóviles de gama } \\
\text { alta. }\end{array}$ \\
\hline 48 & $\begin{array}{l}\text { UNIV. COLEGIO MAYOR DE } \\
\text { CUNDINAMARCA }\end{array}$ & Institución de educación superior. \\
\hline 49 & UNIVERSIDAD EL BOSQUE & Institución de educación superior. \\
\hline 50 & FOLCLOR Y CIENCIA - FYC & $\begin{array}{l}\text { Fundación Promotora de folclor y } \\
\text { ciencia. }\end{array}$ \\
\hline
\end{tabular}

Características de la muestra, figura 1

Número de empleados de las empresas encuestadas

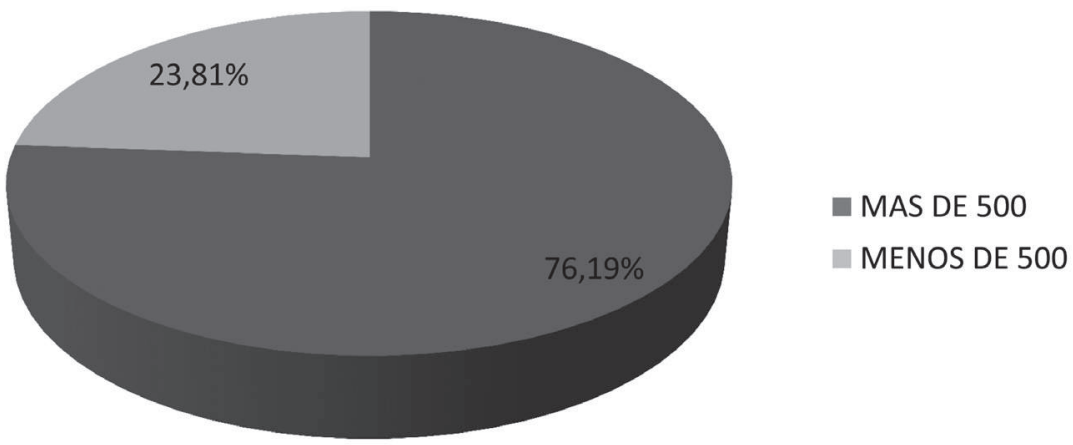

Figura 1. Mas de $50076.19 \%$, Menos de $50023.81 \%$

Diseño metodológico: Estudio cuantitativo, no experimental, descriptivo.

Método: Se utilizó como método de medición la encuesta; las preguntas que se realizaron a las empresas participantes fueron: 
1. ¿Utiliza software libre en la empresa?

2. ¿Se ha evaluado la opción de software libre en la empresa?

3. ¿Hace cuanto utiliza el ampresa software libre?

\section{Resultados}

A la pregunta ¿Utiliza el software libre en la empresa? El $47.62 \%$ respondieron que sí y el $52.38 \%$ que no, Figura 2.

Figura 1. Si $47.62 \% \quad$ No $52.38 \%$

\section{Uso actual del Software Libre en el sector empresarial Bogotano}

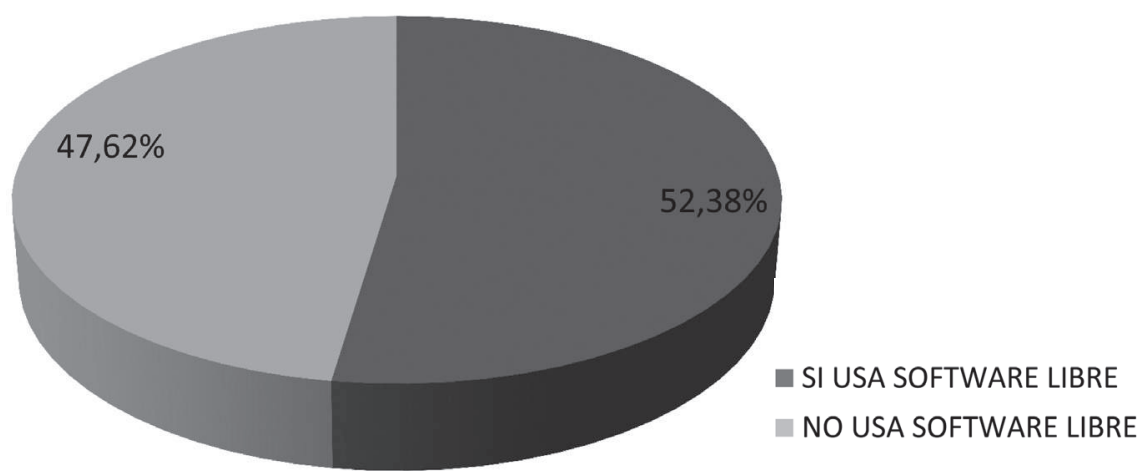

Figura 2. Uso actual del Software Libre en el sector empresarial Colombiano

En la figura 2, se puede apreciar cómo el uso del Software Libre ha ganado un reconocimiento en el sector empresarial ya que es mayor el porcentaje, $52,38 \%$, de las empresas que lo está utilizándo. De acuerdo con los comentarios del formulario se tiene que Linux como sistema operacional, es sin duda, el producto más popular de Software Libre y como consecuencia el más utilizado.

Otro gran grupo de usuarios utiliza Software Libre en aplicaciones de seguridad, y algunos como servidor de Base de Datos en aplicaciones Web.

En la figura 1 se presenta el número de empleados de las empresas participantes donde el 23,81\% son empresas de más de 500 empleados y el 76,19\% de menos, de 500 empleados, y lo que muestra que el instrumento se aplicó tanto en Pymes como grandes empresas. 
Además, se puede apreciar que el uso en pequeñas y medianas empresas que se ubican dentro de menos de 500 empleados es un considerable porcentaje.

A la pregunta: ¿Se ha evaluado la opción del software libre en la empresa?, en igual porcentaje las empresas respondieron haberlo evaluado, haberlo evaluado y descartado y haberlo evaluado con proyección de utilizarlo, Figura 3.

Figura 3: No se ha evaluado, 38.10\%. Se ha evaluado y descartado. $38.10 \%$. Se ha evaluado y hay planes de usarlo, $38.10 \%$

\section{Motivos de las empresas que no utilizan Software Libre}

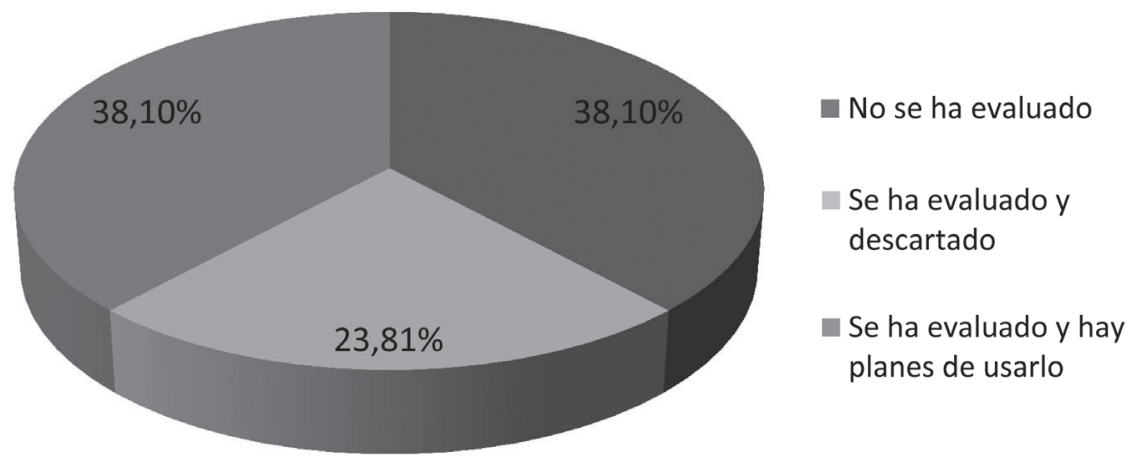

Figura 3. Motivos de las empresas que no utilizan Software Libre

Se puede apreciar cómo el porcentaje de empresas que no han evaluado la utilización del software libre, 38,10\%, y el porcentaje de las empresas que lo han evaluado y tienen planes de usarlo, $38,10 \%$, es igual si se tiene en cuenta la suma de estos dos porcentajes; se obtiene un 76,20\% que es un alto porcentaje frente a las empresas que lo an evaluado y definitivamente lo han descartado, $23,81 \%$.

El grupo de empresas que descartaron el uso del software libre lo hacían porque algunos de sus estándares no eran compatibles o no tenían una asesoría de alguna empresa especializada. Las que no lo utilizan es por falta de conocimiento, lo que hace fundamental el desarrollo de eventos de promoción del Software Libre.

A la pregunta ¿Utiliza la empresa el sofware libre?, el $26.67 \%$ respondio menos de un año, el mismo porcentaje menos de dos años, el 6.67\% menos de tres años y el 40\% mas de cinco años, Figura 4. 
Figura 4. Menos de 1 año. 26.67\% Menos de 2 años. 26.67\% Menos de 3 años. $6.67 \%$ Más de 5 años. $40.00 \%$

\section{Tiempo de uso del Software Libre en el sector Empresarial Bogotano}

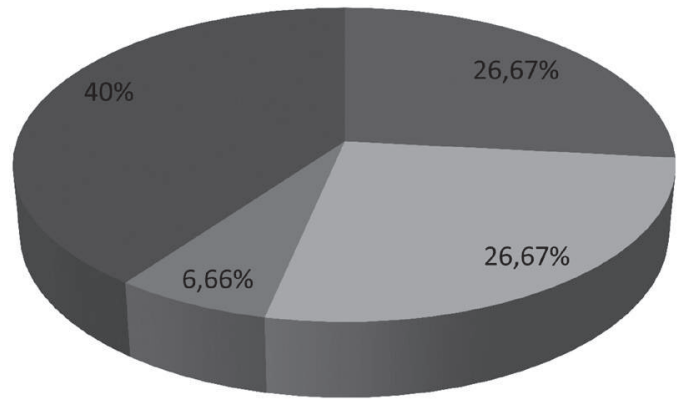

- Menos de 1 año

Menos de 2 años

Menos de 3 años

Mas de 3 años

Figura 4. Tiempo de uso del Software Libre en el sector empresarial

En el anterior gráfico se aprecia que las empresas que vienen utilizando el software libre desde hace menos de dos años $(26,67 \%)$ y desde hace menos de un años $(26,67 \%)$ es del $53,40 \%$, que corresponde a un alto porcentaje de empresas que se han iniciado en el uso del software libre.

\section{Discusión}

Los resultados demuestran que el software libre está listo para su uso y que se viene adoptando con niveles de satisfacción muy altos y grandes resultados. Son muchos los beneficios que genera el uso de software libre, si el gobierno colombiano llegase a promover este uso se puede llegar a disminuir la dependencia de los proveedores de software propietario.

Gran porcentaje de pymes ya hacen uso del software libre; por esto se debe capacitar en las universidades profesionales con capacidades técnicas y de soporte en este tipo de software. Falta apoyo por parte del gobierno colombiano para implementar normas en el uso del software libre, inclusive para genera medidas de apoyo a la capacitación del personal y evaluación del uso de las mismas.

Una de las dificultades al hablar sobre software libre bajo el contexto de cubrimiento del mercado, es la carencia de estadísticas relevantes que soporten las opiniones. No existe la información suficiente, por lo cual es necesario recurrir a las investigaciones realizadas por diferentes universidades, como es el caso de este proyecto de investigación. 
Debido a la ausencia de políticas del uso del software libre en las Universidades colombianas, se ha demorado el uso del software libre; algunos estudiantes y algunos profesores han adoptado el uso pero como iniciativa propia.

El impacto del software libre debería de ser mayor ya que si vemos que en el mundo grandes empresas como IBM, Oracle, Amazon, Yahoo, Google, inclusive la NASA usan y desarrollan software libre.

\section{Referencias bibliográficas}

Bianco, C., Peirano F. (2011). La Brecha Digital en Argentina, Chile y Uruguay. Recuperado http://www3.centroredes.org.ar/files/documentos/Doc.Nro22.pdf

Davila M. (2011). GNU/Linux y el software libre y sus múltiples aplicaciones. Bogotá, Colombia: Universidad Minuto de Dios. Alfaomega Grupo Editor.

Delgado J., Paz F. (2012). Guía Práctica Open Office.org 3.0 Bogotá, Colombia: Editorial Anaya.

García M. (2011). Sobre el Software Libre. Badajoz, España. Recuperado: http://www. cenatic.es/sobre-el-software-libre.

Gates, W. (2012). An open letter to hobbyists. General Partner, Micro-Soft. Recuperado http://www.blinkenlights.com/classiccmp/gateswhine.html

Gonzalez, J., Seoane, J., Robles Gregorio. (2011). Introducción al Software libre. Madrid, España. Universidad Rey Juan Carlos.

Meizoso L. (2012). Open Source Software Usage by European Public Administrations. Madrid, España. Recuperado: http://www.mentatconsultores.com/?s=software+libre \&searchsubmit=

Stallman, R. (2012). Software libre para una sociedad libre. Recuperado http://stallman.org/ Suarez, M. (2011). El software libre y la Administración Pública. Caracas, Venezuela. Ministerio de Ciencia y Tecnología. 